\title{
Ausbildungsinstitut für Klinische Verhaltenstherapie (AFKV in NRW)
}

Das AFKV, 1987 von Psychologen und Ärzten gegründet, bietet ein von der Kassenärztlichen Bundesvereinigung, dem Landesministerium für Arbeit, Gesundheit und Soziales sowie vom Deutschen Fachverband für Verhaltenstherapie (DVT) anerkanntes Ausbildungskonzept. Das Ausbildungsinstitut für Klinische Verhaltenstherapie (AFKV) ist eine gemeinnützig anerk. Einrichtung. Gewinne werden in Forschung und Aus-bildungsförderung investiert. Bausteine der Ausbildung werden auch von Ärztekammern anerkannt.

Die theoretische und praktische Ausbildung zum Psycholo-gischen Psychotherapeuten erfolgt in 8 Semestern berufsbe-gleitend, in der Regel wöchentlich einmal am Abend und an Samstagen. Die Selbsterfahrung ist fester Bestandteil. Supervision und Einzelselbsterfahrung erfolgen durch KBV-anerk. Institutssupervisoren.

Nach der Zwischenprüfung (3./4. Semester) und weiteren Vor-aussetzungen kann im Beauftragungsverfahren zu Lasten der Krankenkassen unter Supervision gearbeitet werden. Nach Abschluß der Ausbildung (8 Semester Theorie, Selbsterfahrung, Fallarbeit unter Supervision und Abschlußprüfung sowie

Absolvierung des Klinischen Jahres) kann zur Ausübung der Verhaltenstherapie nach den Psychotherapie $\pi^{\prime} \mathrm{cM} / \mathrm{mm}$ und -vereinbarungen die Zulassung zu den Krankenkassen bean-tragt werden.

Im Hinblick auf das zu erwartende Psychotherapeutengesetz sind Approbation und sozialrechtliche Zulassungen auf Grund der Ausbildungsnachweise zu erwarten.

Aktuelle Aus- und Weiterbildungsangebote

Beginn eines VT-Ausbildungskurses jeweils im August/September; pro Semester ca. 40-110 Unterrichtseinheiten einschließ-lich Gruppenselbsterfahrung. Beginn der praktischen Fallarbeit mit Mentoren- und Supervisionshilfe ab dem 1./2. Semester. Jährliche Sonderveranstaltungen mit renommierten Hochschullehrern (Prof. Hautzinger, Prof. Kanfer, Prof. Schulte u. a.). Seit 1991 bietet das AFKV außerdem eine Zusatzausbildung in Kinder- und Jugendlichen-VT an. Diese ebenfalls KBV-anerk. Ausbildung kann wegen der in 11 Blöcken (120 Stunden Theorie über 1 Jahr an Wochenenden und Feiertagen stattfindenden Terminen auch parallel zur VT-Hauptausbildung absolviert werden.

Über Sonderteilnahme entscheidet der Ausbildungsausschuß. Beginn jeweils im Oktober. 
Nach weitgehender Festlegung der Übergangsregelung zum Psychotherapeutengesetz besteht die Möglichkeit zur Teil-nahme an einem AufbauErgänzungskurs mit Ausbildungsbau-steinen, insbesondere Praxis-bezogenen Lehrveranstaltungen an Wochenenden mit Einzelbausteinen. Beginn Februar 1997. Für Ärzte ist eine Selbsterfahrungsgruppe unter ärztlicher Leitung vorgesehen.

Am 18. Oktober 97 veranstaltet das AFKV aus Anlaß des 1Ojährigen Bestehens einen wissenschaftlichen Tag mit dem Thema: Verhaltenstherapie Entwicklungslinien und Per-spektiven unter Teilnahme von Prof. Grawe, Prof. Schulte, Prof. Reinecker u.a.

Ausführliche Informationshefte erhalten Sie bei schriftlicher Anfrage über das Sekretariat (frankierter DIN-A5-Umschlag). Telef. Sprechzeiten vor-mittags von 9 bis $11 \mathrm{~h}$.

AFKV Sekretariat und Geschäftsstelle: Cranger Straße 129 D-45891 Gelsenkirchen Tel. 02 09/7 6490 Fax 02 09/78 0499

Arbeitsgemeinschaft für Verhaltensmodifíkation e.V. (AVM-D)

Die ordentliche Mitgliederversammlung fand am 27. April in

Bamberg statt. Neben den Berichten des Vorstandes, der

Awank, wurde der Bericht des Kassiers entgegengenommen, der Kassenbericht genehmigt und der Vorstand entlastet. Im Mittelpunkt der MV stand die geplante Änderung der Ausbildungsordnung. Der Vorstand wurde beauftragt, diese Arbeit fortzusetzen. Die Ergebnisse sollen in einer außerordentlichen Mitgliederversammlung am 23. November 1996 vorgelegt werden. Am gleichen Tag, vor Beginn der MV, wird eine AK-Leiter- und Lehrtherapeuten-Konferenz stattfinden. Bei der AK-Leiter- und Lehrtherapeuten-Konferenz am 30.3.96 wurde Herr Dr. Rudi Wagner als Lehrtherapeut anerkannt.

An dem Treffen der AVM-Österreich und der AVM-Schweiz nahmen drei unserer Vorstandsmitglieder teil.

Die von uns durch eine Anschubfinanzierung unterstützte Psychologische Forschungs- und Beratungsstelle (PFB) in Würzburg arbeitet unter der akademischen Leitung von Herrn Prof. Dr. Heiner Ellgring. Die organisatorische Leitung hat Herr Dipl.-Psych. Ralph Schwarz, als Projektmitarbeiter ist Herr Dr. Dipl.-Psych. Michael Macht angestellt.

Im Rahmen der Ausbildung zum Verhaltenstherapeuten arbeiten in der PFB zehn Diplom-Psychologen mit ca. zwanzig 


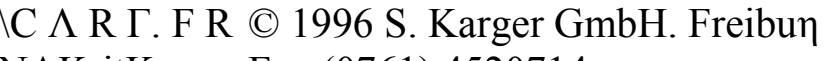
NAK $<j$ tK $\quad$ Fax (0761) 4520714 
Klienten. Neun der Therapeuten befinden sich in der Ausbil-dung der AVM.

Schwerpunkte sind die angewandte Forschung im Bereich der Interventionspsychologie, die Aus- und Weiterbildung in der Verhaltenstherapie, die

Intervention im Rahmen der Forschung oder der verhaltenstherapeutischen Ausbildung. Von der Deutschen Parkinson-Vereinigung wird der PFB eine halbe Psychologenstelle finanziert mit dem Ziel, eine psycho-logische Parkinson-Ambulanz für den Raum Franken, Nord-baden und Südhessen aufzubauen. Mit der Entwicklung eines Manuals sollen Psychotherapeuten Möglichkeiten zur Intervention bei Morbus Parkinson aufgezeigt werden. Die Supervisor/innen sind zu einem Workshop mit Dr. Maren Langlotz-Weis am 29./30.11. in Würzburg geladen.

AVM-Geschäftsstelle:

Lehrstuhl für klinische Psychologie c/o Frau Dagmar Frey Universität Bamberg Postfachl549 D-96045 Bamberg

Institut für Fort- und Weiterbildung in Klinischer Verhaltenstherapie (IFKV)

Das IFKV, Bad Dürkheim, bietet wiederum ab Frühjahr 1997

an:

Ausbildung zum Supervisor, Schwerpunkt Verhaltenstherapie.

Theorie:

Allgemeine versus verhaltenstherapeutische Supervision

Techniken der Supervision

Supervisionsmodelle verschiedener Therapieschulen

Organisations- und Teamsupervision

Entwicklungsprozesse in der Supervision Praxis:

Praxisseminare

Supervision der Supervision durch Lehrtherapeuten

Co-Supervision und Intervision

Anmeldung zum Info-Gespräch am 27.11.96 und weitere Auskünfte:

IFKV-Sekretariat

Kurbrunnenstraße 21a

67098 Bad Dürkheim

Tel. (06322)68019

Fax (06322) 68010

Vereinigung der Kassenpsychotherapeuten

Im Juni 1996 übergab die Vereinigung der Kassenpsychotherapeuten der KBV und den Krankenkassen ein Konzept für eine Revision der Kapitel G IV und G

V des EBM. Dieses Konzept soil dazu beitragen, die Abschnitte Psychotherapie und Test-diagnostik endlich den realen Gegebenheiten anzupassen und die

Arbeit zu erleichtern. Benachteiligungen der Psychologi- 
schen Psychotherapeuten gegenüber den ärztlichen Psychotherapeuten sollen aufgehoben und die negativen Erfahrun-gen mit dem neuen EBM sollen berücksichtigt werden.

Grundsätze einer EBM-Revision des Kapitels G IV (Psychotherapie)

Vorbemerkungen

In Hinblick auf das bevorstehende Psychotherapeutengesetz und die mehrheitlich gewünschte Integration der Psychologi-schen Psychotherapeuten in das System der vertragsärztlichen Versorgung müssen die im Integrationsmodell niedergelegten Grundsätze der gleichberechtigten Behandlung ärztlicher und psychologischer Psychotherapeuten auch bei der EBM-Re-form des Kapitels G IV gewahrt bleiben. Psychotherapie bleibt daher ein Leistungsbereich, der innerhalb der vertragsärztlichen Versorgung entsprechend den Psychotherapierichtlimen in einem eigenen Abschnitt des EBM zu regeln ist. Die hier beschriebenen Leistungen müssen sowohl durch ärztliche Psychotherapeuten wie auch durch Psycholo-gische Psychotherapeuten und Kinder- und Jugendlichen-psychotherapeuten erbracht werden können. Dies setzt voraus, daß psychotherapeutische Leistungen klar von solchen Leistungen abzugrenzen sind, deren Erbringung nicht durch die Erfordernisse der Psychotherapierichtlimen geregelt ist und die einer anders definierten Qualifikation bedürfen. Nur auf diese Weise kann den fachlichen und ver-sorgungsbezogenen Besonderheiten der Psychotherapie ent-sprochen werden.

Bei der Neugestaltung des Kapitels G IV ist ferner darauf zu achten, daß künftig auch Leistungen der Prävention und Rehabilitation berücksichtigt werden sollten. Ebenso sollten spezi-fische Beratungsleistungen vorgesehen werden, die nur durch Psychotherapeuten erbracht werden können. Die notwendige Zusammenarbeit zwischen Psychologischen Psychotherapeuten und den ärztlichen Kollegen aller Fachge-biete ist nach Maßgabe des Konsiliarverfahrens auch im EBM gebührentechnisch zu regeln. Davon unberührt bleibt das Recht des Patienten auf die freie Wahl des Behandlers und die damit verbundene Möglichkeit des Erstzugangs auch zum Psychologischen Psychotherapeuten.

Eine Überarbeitung des Kapitels G IV sollte unserer Meinung nach von folgenden Grundsätzen ausgehen:

Breiteres Abrechnungsspektrum: Für die leistungsgerechte und wirtschaftliche Psychotherapie wird ein Spektrum von Gebührenordnungspositionen benötigt, das den Leistungs-bedarf, der ohnehin schon erbracht wird, abdeckt. In der Vergangenheit mußten Leistungen, die in der psychothera-peutischen Praxis anfallen, entweder in die wenigen zur Verfügung stehenden Ziffern gezwängt werden, oder sie konnten nicht abgerechnet werden.

Mengenbegrenzung: Es sollten keine Gebührenordnungspositionen geschaffen oder zugänglich gemacht werden, die 

Mitteilungen der Verbände

Information by Behavior Therapy Associations 
-Psychotherapie in Gruppen: Ein Leistungsbereich, dessen unzureichende Regelung sowohl im EBM als auch in den Psychotherapierichtlinien sich bisher besonders hemmend auswirkte, ist die Gruppenpsychotherapie. Die Gründe sind kurz zusammengefaßt:

-die entmutigend hohen bürokratischen Hürden bei der Antragsstellung,

die ungünstige Kontingenzanrechnung der Gruppen- und Einzeltherapiestunden,

die unzureichende Honorierung, die auch durch den neuen EBM nicht attraktiver wurde.

Dies führte letztlich dazu, daß gruppentherapeutisches Arbeiten in der ambulanten Praxis fast völlig aufgegeben wurde, während in der stationären

Psychotherapie vorwie-gend gruppentherapeutisch gearbeitet wird. Somit entstand ein letztlich kostenträchtiger Wettbewerbsvorteil der stationären gegenüber der ambulanten Therapie. Durch die nicht antragspflichtigen gruppentherapeutischen Möglichkeiten in der kleinen EBM-Reform für Psychiater ist hier ein weiterer Wettbewerbsnachteil für die Psychotherapeuten entstanden. Vorschläge, wie Gruppentherapie in den Psychotherapierichtlinien günstiger gestaltet werden könnte, haben wir be-reits an anderer Stelle unterbreitet. Was den EBM betrifft, sind zwei Maßnahmen erforderlich:

relative Höherbewertung im Verhältnis zur Einzeltherapie (die Schwelle der Teifnehmerzahl, ab der Gruppentherapie lohnender ist als Einzeltherapie, liegt auch im neuen EBM bei 6 Patienten)

die Förderung von Kleingruppen mit bis zu 5 Teilnehmern für spezielle verhaltenstherapeutische Expositionsbehand-lungen durch eigene, höher bewertete Gebührenordnungs-positionen für solche Gruppen.

Psychotherapie bei Kíndern und Jugendlíchen: Das bisherige Leistungsspektrum des Kapitels G IV ist auf die Psychotherapie mit Erwachsenen zugeschnitten. In der Psychotherapie mit Kindern und Jugendlichen besteht ein erhöhter Bedarf an begleitenden Maßnahmen und Abstimmungen mit den Bezugspersonen, oft außerhalb der Praxisräume. Das Leistungsspektrum sollte um entsprechende Ziffern erweitert werden.

Grundleistungen: Psychologische Psychotherapeuten und Kinder- und Jugendlichenpsychotherapeuten benötigen weitere Abrechnungsmöglichkeiten für Grundleistungen, insbesondere für:

den Verwaltungsaufwand analog zur Ordinationsgebühr. Wegen der geringen Fallzahlen (max. 50-70) und des relativ höheren Verwaltungsaufwandes sollte diese Ziffer psychotherapiespezifisch definiert und höher bewertet werden als die Ziffer 1.

Telefonate zwischen Patient und Therapeut, wie sie z. B. in der Verhaltenstherapie zur Rückmeldung über Übungen und Hausaufgaben notwendig sind. Mit der Ordinationsgebühr oder der Konsultationsgebühr ist dies nur ungenü-gend erfaßt.

zu einer unkontrollierten Mengenausdehnung verführen. Dies garantieren können am ehesten Ziffern, die

an Zeitvorgaben gebunden sind,

präzise definiert sind, und

deren Abrechnung vom Patienten nachvollzogen werden kann.

Erst alle drei Kriterien zusammen gewährleisten die Kon-trolle der Mengenentwicklung. Die Erfahrung im ersten Quartal 1996 beispielsweise mit den Ziffern 10,11 und 17 hat gezeigt, daß Zeitvorgaben allein nicht ausreichen. Diese Kriterien sichern auch die Qualität der Psychotherapie, weil das Leistungsspektrum sich nicht an der Abrechnung orientieren würde. Ein weiterer psychotherapiespezifischer Gesichtspunkt spielt hierbei eine Rolle: Psychotherapie er-fordert eine Behandlungsatmosphäre, die ruhiges Nachden-ken und -forschen ermöglicht. Eine abrechnungstechnisch bedingte Hektik im Praxisablauf ware kontraproduktiv.

- Feste Punktwerte: Durch die Zeitvorgaben psychotherapeu-tischer Leistungen ist eine Erhöhung des Honorars nur in einem beschränkten Rahmen möglich. Ein Schutz vor der finanziellen Aushöhlung dieses Bereiches durch andere Lei-stungsbereiche kann nur durch feste oder nach unten 1·imi-tierte Punktwerte garantiert werden. Auch eine Zunahme der Behandlerzahl darf sich nicht negativ auf die Honor are auswirken, solange es im Bereich Psychotherapie keine Bedarfsplanung und keine Zulassungsbeschränkung gibt.

Die im folgenden aufgeführten Vorschläge erfordern flankie-

rend auch eine Änderung der Psychotherapierichtlinien.

-Flexiblere Zeiteinheiten: Kernbereich der Psychotherapie müssen die zeitgebundenen psychotherapeutischen Sitzungen bleiben. Hier könnten aber flexiblere Lösungen diskutiert werden. Die relativ starre Bindung an 50-Minuten-Einheiten ist eine fachlich nicht begründete Tradition. Daß sich inzwi-schen die meisten Therapeuten diesem Stundenrhythmus angepaßt haben, ist keine Rechtfertigung für ihre Beibehal-tung. Je nach Störung oder Patient sind unterschiedlich lange Sitzungen erforderlich.

Dabei muß jedoch berücksichtigt werden, daß zur Entste-

hung einer vertrauensvollen Beziehung in der Psychothera

pie auch eine gewisse Konstanz der Rahmenbedingungen er

forderlich ist. -

-Niederfrequente, nicht antragspflichtige Psychotherapie: In einer psychotherapeutischen Praxis werden auch Patienten behandelt, die eine langfristige psychotherapeutische Unter-stützung, oft in niederfrequenter Form, benötigen. Bei diesen chronisch psychosomatisch oder psychotisch kranken Patienten ist eine zeitlich befristete, antrags- und oft gutachter-pflichtige Therapie, wie sie die Psychotherapierichtlinien alleine vorsehen, nicht angemessen.

-Krisenintervention: Manche Krankenhauseinweisung könnte vermieden werden, wenn eine sofortige psychotherapeutische, antragsfreie Krisenintervention möglich ware. Entsprechende Leistungspositionen sollten von der regulären, antragspflich-tigen Psychotherapie definitorisch abgegrenzt sein. 
Mitteilungen der Verbände

Information by Behavior Therapy Associations 
Berichte zur Verbesserung der Koordination zwischen Psychotherapeut und Arzt.

Konsiliarische Erörterungen.

Besuche von Patienten, die aus Krankheitsgründen nicht in der Praxis erscheinen können.

Vorschlag f ür die Neuordnung des Kapitels G V des EBM (Testverfahren)

Testdiagnostik ist psychotherapeutisch relevant:

- $\quad$ zur Objektivierung der Diagnose zu Beginn einer Therapie.

Die Zieldefinition und die Entscheidung für einen Behand-

lungsplan können bei manchen Patienten präziser getroffen

werden, z. B. bei einer differentialdiagnostischen Abklärung

einer intellektuellen Beeinträchigung, einer Psychose, einer

Persönlichkeitsstörung,

-zur Objektivierung des Therapieerfolges durch Vor ${ }^{-}$, Zwischen- und Nachuntersuchungen,

als Rückmeldung für den Patienten mit dem Ziel einer rea-listischeren Selbsteinschätzung,

als Teil der Basisdokumentation für wissenschaftliche Zwecke.

Bisher wurden Testverfahren wegen der geringen Bewertung im EBM und wegen der durch die Psychotherapierichtlinien vorgegebenen Beschränkungen selten im psychotherapeu-tischen Alltag eingesetzt.

Bei der Neuordnung und Neubewertung der Testverfahren gehen wir von folgenden Überlegungen aus:

1. Qualifikationsvoraussetzungen

Während die Testdurchführung bei einem Teil der Tests auch von Hilfskräften vorgenommen werden oder apparativ erfol-gen kann, ist die Testínterpretation immer von dafür speziell ausgebildeten Ärzten, Psychologischen Psychotherapeuten und Kinder- und Jugendlichenpsychotherapeuten durchzuführen. Die spezielle Qualifikation umfaßt ein Wissen über Test-theorie, Testaufbau, Testentwicklung, Testdurchführung und Testinterpretation [s. Lienert, 1969].

Insbesondere ist ein fun-diertes Wissen über Gütekriterien und Grenzen eines Tests er-forderlich. Eine unkritische Testanwendung kann gravierende

Konsequenzen haben. Welche suggestiven Auswirkungen dia-gnostische Falschaussagen haben können, zeigt eindrucksvoll ein berühmt gewordenes Experiment von Rosenthal [Rosen-thai und Jacobson, 1974].

Eine unqualifizierte Testanwendung ist ebensowenig zu ver-treten wie eine unqualifizierte Röntgendiagnostik. Aus diesem Grund ist es notwendig, Testdiagnostik in die Psychotherapierichtlinien aufzunehmen und dort die Qualifikationsvoraussetzungen zu definieren.

2. Zeitaufwand und Bewertung

Bei der Berechnung des Zeitaufwandes müssen Testvorberei-tung, Testdurchführung, Testinterpretation sowie Material- 
kosten berücksichtigt werden. Außerdem ist zu berücksich-tigen, zu welchen Anteilen die Testdurchführung von einer Hilfsperson vorgenommen werden kann oder nicht. In den jeweiligen Testmanualen ist der Zeitaufwand einfach festzustellen. Da in Deutschland mindestens 100 klinisch rele-vante Tests auf dem Markt sind, ware es nicht sinnvoll, sie alle im EBM aufzuführen. Beispielhaft könnten zu jeder der vor-geschlagenen Kategorien einige der gängigsten Tests erwähnt werden. Der Behandler könnte die jeweils zutreffende Ziffer anhand des im Manual genannten Aufwandes selbst bestim-men. Die Korrektheit der Abrechnung läßt sich einfach über-prüfen, wenn die Abkürzung des angewandten Tests hinter der Abrechnungsziffer vermerkt wird.

Da es sich um zeitgebundene Leistungen handelt, sollte sich die Bewertung der Testverfahren an der Bewertung psycho-therapeutischer Leistungen orientieren, d.h., ein Test, der einen Aufwand von 60 min von der Durchführung bis zur Interpretation und Dokumentation erfordert, würde etwa einer Psychotherapiesitzung von 50 min Dauer entsprechen (1450 Punkte). Die Gewichtung der Bewertung mit dem Faktor «Durchführung durch Hilfskraft oder Apparate» müßte für jeden Test einzeln bestimmt werden.

3. Anwendungsmöglichkeit

Psychodiagnostik unterstützt den therapeutischen Prozeß, wenn sie therapiebegleitend verstanden wird. Anders als in den Psychotherapierichtlinien festgelegt, sollten Tests deshalb in jeder Phase einer Therapie angewendet werden können.

Testverfahren lassen sich nach unterschiedlichen Kriterien klassifizieren [s. Brickenkamp, 1975]. Wir stellen drei mögliche

Systeme vor.

Die von Brickenkamp selbst entwickelte und allgemein aner-

kannte Klassifikation unterscheidet:

1. Leistungstests

Entwicklungstests, Intelligenztests, Allgemeine Leistungstests, Schultests, Spezielle Funktionsprüfungs- u. Eignungs-tests Psychometrische Persönlichkeitstests Persönlichkeits-Strukturtests, Einstell·ungs- und Interessen-tests, Klinische Tests

Persönlichkeits-Entfaltungsverfahren («projektive Ver-fahren»).

Formdeuteverfahren, verbal-thematische Verfahren, zeich-

nerische u. Gestaltungsverfahren. Brickenkamp rechtfertigt die Umbenennung der projektiven Verfahren in «Persönlichkeits-Entfaltungsverfahren» mit der Mehrdeutigkeit und Problematik des Begriffes «projektiv». In seinem Standardwerk wird jedes Testverfahren genau beschrieben und einer kritischen Würdigung unterzogen (s. einige Beispiele als Anlage). In einem Ergänzungsband, der 1983 erschienen ist, wurden neuere Testverfahren auf-genommen. Es soil 1997 eine neue und aktualisierte Auflage erscheinen. 

Mitteilungen der Verbände

Information by Behavior Therapy Associations 
Im Leistungsverzeichnis der TK-Regelung wird eine Einteilung nach Intelligenztests,

Leistungstests,

Persönlichkeitstests

vorgenommen. Sachlich problematisch ist hier die Unterschei-dung zwischen Intelligenztests und Leistungstests als zwei ge-trennte Hauptkategorien. Im EBM wird unterschieden zwischen:

Fragebogentests,

Orientierenden Testverfahren,

Funktionstests,

Projektiven Testverfahren,

Intelligenz- und Entwicklungstests,

Aufwendigen projektiven Testverfahren.

Diese Systematik ist in inhaltlicher Hinsicht unlogisch: Zwischen 2. und 3. gibt es keinen inhaltlichen Unterschied, zwischen 4. und 6. ebenfalls nicht. Kategorie 2 enthält sowohl projektive Verfahren, für die es zwei weitere Kategorien (4. und 5.) gibt, als auch Intelligenztests. Einige der beispiel-haft aufgeführten Verfahren sind veraltet und möglicherweise auch im Handel nicht mehr erhältlich. Die EBM-Klassifika-tion scheint sich nicht nach inhaltlichen Kriterien, sondern nach dem erforderlichen Zeitaufwand zu richten. Unserer Meinung nach sollten beide Kriterien, Inhalt und Zeitaufwand, Berücksichtigung finden.

Die Berücksichtigung des Zeitaufwandes, unter Einberech-nung einer eventuellen apparativen oder hilfskraftunterstütz-ten Durchführung, spielt bei der EBMrelevanten Einteilung eine zentrale Rolle und muß bei einer Punktzahl-Bewertung kalkulatorisch zugrundegelegt werden. Sinnvoll ist eine gene-relle Einordnung der Verfahren nach Zeitaufwand, wie sie etwa in der TK-Regelung vorgenommen wird:

geringer Aufwand (mind. $30 \mathrm{~min}$ ),

mittlerer Aufwand (mind. $60 \mathrm{~min}$ ),

hoher Aufwand (mind. $120 \mathrm{~min}$ )

Nicht nachvollziehbar ist bei dieser Unterteilung der zeitliche Sprung zwischen mittlerem und hohem Aufwand.

Schlußfolgerung

Werden beide Einteilungen, die inhaltliche von Brickenkamp und die zeitbezogene, wie hier vorgeschlagen kombiniert,

würden 12 EBM-Ziffern ausreichen, das Feld Testverfahren hinlänglich differenziert nach inhaltlichen und Zeitkriterien abzubilden:

Leistungstests Psychometrische Projektive Verfahren

Persönlichkeitstests

Mind. 30 min (1) (2) (3)

Mind. $60 \mathrm{~min} \quad$ (4) (5) (6)

Mind. $90 \mathrm{~min} \quad$ (7) (8) (9)

Mind. $120 \mathrm{~min} \quad(10)(11) \quad(12)$ 
Hier erwähnte und weiterführende Literatur

Berufsverband Deutscher Psychologen: TK-Regelung, Bonn, 1994.

Brickenkamp R: Handbuch psychologischer und pädagogischer Tests.

Göttingen, Hogrefe, 1975.

Brickenkamp R: Erster Ergänzungsband zum Handbuch psychologischer

und pädagogischer Tests. Göttingen, Hogrefe, 1983.

Jäger R, Petermann F: Psychologische Diagnostik. München, Psychologie

Verlags Union, 1975.

Lienert GA: Testaufbau und Testanalyse. Weinheim, Beltz, 1969.

Rauchfleisch U: Psychodiagnostik in der psychoanalytisch orientierten

Psychotherapie; in: Psychotherapeut 1996, 41, pp 69-76.

Rosenthal R, Jacobson L: Pygmalion im Klassenzimmer. Weinheim, Beltz,

1974.

Stieglitz RD, Baumann U: Psychodiagnostik psychischer Störungen.

Stuttgart, Enke, 1994.

Schmidtchen S: Psychologische Tests.

Geschäftssteüe der

Vereinigung der Kassenpsychotherapeuten:

Dipl.-Psych. Dieter Best

Riedsaumstraße 4a

67063 Ludwigshafen

Tel. (0621)637015

Fax (0621) 637016

Verein zur Förderung der klinischen Verhaltenstherapie e.V. (VFKV)

Der Verein zur Förderung der klinischen Verhaltenstherapie wurde am 22.3.1983 gegründet. Besonderes Ziel war die Eröffnung des 1.

Weiterbildungsinstituts, das eine curriculare, mehrsemestrige, berufsbegleitende Ausbildung in Verhaltenstherapie bietet. Inzwischen kann eine 3- bzw.

5jährige Weiter-bildung absolviert werden, ebenso die Ausbildung für verhal-tenstherapeutische Gruppentherapie und in naher Zukunft Verhaltenstherapie für Kinder und Jugendliche. Das Institut ist von der Kassenärztlichen Bundesvereinigung anerkannt, die Curricula entsprechen den jeweiligen Richt-linien.

Die Ausbildungsjahrgänge beginnen jeweils im Herbst mit 15 Psychologen und Ärzten. Bis zum Herbst 95 wurden 13 Ausbildungsgänge durchgeführt.

Der Verein arbeitet auf der Grundlage einer Satzung und ist gemeinnützig. Er wird von einem aus 6 Mitgliedern bestehen-den Vorstand geleitet, wobei die

Positionen des 1. und 2. Vor-sitzenden paritätisch von Ärzten/Psychologen besetzt sein müssen. Außer der Weiterbildung werden wissenschaftliche Projekte gefördert. Überschüsse werden entweder in therapie-bezogene Forschungsprojekte investiert und/oder für Ausbil-dungsstipendien an solche Teilnehmer verwendet, die sich sonst die Ausbildung nicht leisten könnten. Der Verein hat einen Geschäftsführer, der auch für organisa-torische Fragen und Beratung von Teilnehmern zur Verfügung steht.

Gegenwärtig werden im Institut zwei parallel laufende Gruppen von jeweils 15 Teilnehmern ausgebildet. 

Mitteilungen der Verbände

Information by Behavior Therapy Associations 
Zum Institut gehört eine verhaltensmedizinische Ambulanz mit zwei Therapieräumen. Patienten werden von niederge-lassenen Ärzten und Beratungsstellen überwiesen. Sie werden im Rahmen eines Erstberatungsgesprächs von einem Beauf-tragungsarzt untersucht und den Ausbildungsteilnehmern zugewiesen. Gegenwärtig wird die Ambulanz von ca. 20 Patienten wahrgenommen. Damit haben alle Ausbildungsteil-nehmer die Möglichkeit, die erforderlichen Behandlungsfälle im Rahmen der Ambulanz zu behandeln. Die Behandlung ist, abgesehen von den Beauftragungsfällen, kostenlos. Im Rahmen der DVTTagung am 1. und 2. März 1996 fanden einige Veranstaltungen in den Räumen des Instituts statt. Als Eigenveranstaltung des Instituts führte Herr Professor Kanfer einen Workshop für Supervisoren durch.

Dr. med. Thomas Utz 1. Vorsitzender d. VFKV

Dipl.-Psych. Walter Hauke 2. Vorsitzender d. VFKV

Dr. med. Dieter Schwarz, Geschäftsführer d. VFKV

Geschäftsstelle: VFKV

Pippinger Straße 2581245 München Tel. 089/8346900 Fax 089/8 348659

Fortbildungsinstitut für

klinische Verhaltenstherapie e.V. (FIKV)

10 Jahre Fortbildung in Klinischer Verhaltenstherapie in Bad Pyrmont

1997 besteht das Fortbildungsinstitut für Klinische Verhaltenstherapie (FIKV) Bad Pyrmont im 10. Jahr. In diesen 10 Jahren seines Bestehens hat das Institut sich im südlichen Niedersach-sen und weit darüber hinaus um die Weiterbildung von jungen Ärzten und Diplom-Psychologen bemüht, indem es entspre- 
chende Ausbildungscurricula für Ärzte und Psychologen ange-boten hat und auch weiterhin anbietet.

Weiterbildung für Ärzte und Diplom-Psychologen Das FIKV bietet Möglichkeiten des Erwerbs von Bausteinen für die Fachärzte für Psychiatrie und Psychotherapie, den Fach-arzt für Psychotherapeutische Medizin und für das Führen der Zusatzbezeichnung Psychotherapie für Ärzte anderer Fachrichtungen. Weiterhin besteht die Möglichkeit, Voraussetzun-gen für die psychosomatische Grundversorgung zu erwerben. Für Diplom-Psychologen bietet das FIKV in Zusammenarbeit mit der Psychosomatischen Fachklinik Bad Pyrmont eine 3jährige ganztägige Ausbildung in Verhaltenstherapie an. Zudem findet in regelmäßigen Abständen eine 5jährige be-rufsbegleitende Ausbildung in Verhaltenstherapie für Diplom-Psychologen statt.

Offene Weiterbildungsangebote

Zusätzlich ist es auch Anliegen des Fortbildungsinstitutes für Klinische Verhaltenstherapie, die Akzeptanz und Verbreitung von Verhaltenstherapie zu fördern und verhaltenstherapeuti-sche und verhaltensmedizinisches Denken bekannt zu machen. Aus diesem Grund ist der dritte Weiterbildungsbaustein des

Fortbildungsinstitutes für Klinische Verhaltenstherapie die Durchführung offener Weiterbildungsveranstaltungen, die interessierten Kolleginnen und Kollegen aus dem näheren und weiteren Umkreis eine unverbindliche Kontaktaufnahme mit der Verhaltenstherapie ermöglicht.

«Jubiläums»-Programm 1997

Um sich selber und auch die vielen Interessenten anläßlich des 1Ojährigen Bestehens zu belohnen, hat sich das FIKV für das Jahr 1997 bemüht, mit einem hochkarätigen Programm an Weiterbildungsveranstaltungen inhaltlich und wissenschaftlich das «lOjährige» zu begehen. Unter den Namen der insgesamt 6 Dozenten für das Jahr 1997 finden sich viele namhafte Internationale Vertreter aus dem Bereich der Psychiatrie und Psychotherapie. 

Das Weiterbildungsprogramm 1997

Thema 

Der Selbstmanagementansatz

Verhaltenstherapie bei Angststörungen

Multimodale Verhaltenstherapie bei posttraumatischen Belastungsreaktionen

Partner- und Familientherapie

Verhaltenstherapie bei chronisch psychisch kranken Menschen

Kognitive Verhaltenstherapie bei Depressionen

Anmeldung und weitere Information:

Fortbildungsinstitut für Klinische Verhaltenstherapie, FIKV e.V. 
Prof. Dr. phil. FH. Kanfer, Department of Psychology University 25./26.1.97 of Illinois, USA

Prof. Dr. med. I. Hand, Psychiatrische Universitäts-Klinik 1./2.3.97

Eppendorf, Hamburg

Dr. phil. R. Klepsch, Institut für Gesundheitspsychologie, Hamburg ～19./20.4.97

Prof. Dr. med. J. Willi, Psychiatrische Poliklinik, $\quad 27728.9 .97$

Universitätsspital Zurich

Prof. Dr. rer. nat. A. Kuhr, Arbeitsbereich Klinische Psychologie der 8./9.11.97 Medizinischen Hochschule Hannover Prof. Dr. phil. M. Hautzinger. Psychologisches Institut, $\quad 677.12 .97$

Universität Mainz

Bombergallee 11 — 31812 Bad Pyrmont · Tel. 05281/606763 

Mitteilungen der Verbände

Information by Behavior Therapy Associations 
